\title{
Optimal Wavelet Filters Selection for Ultrasound and Mammography Compression
}

\author{
Volodymyr Ponomaryov, Jose Luis. Sanchez-Ramirez, \\ and Cristina Juarez-Landin \\ National Polytechnic Institute of Mexico, Av. Santana \# 1000, \\ Col. San Fco. Culhuacan, 04430, Mexico, D.F. Mexico \\ vponomar@mail.ru, jluissar@yahoo.com.mx, cjlandin@yahoo.com.mx
}

\begin{abstract}
The analysis of different Wavelets also including novel Wavelets families based on atomic functions are presented, especially for Ultrasound (US) and Mammography (MG) images compression. This way we have been able to determine what type of filters Wavelet works better in compression of such images. Key properties: Frequency response, Approximation Order, Projection cosine, and Riesz bounds were determined and compared for the classic Wavelets W9/7 used in standard JPEG2000, Daubechies8, Symlet8, as well as for the complex Kravchenko-Rvachev wavelets $\psi(\mathrm{t})$ based on the Atomic Functions $u p(t), f u p_{2}(t)$, and eup(t). The comparison results show significantly better performance of novel Wavelets that is justified by experiments and in study of key properties.
\end{abstract}

Keywords: Wavelet Transform, Compression, Atomic Functions, Ultrasound images, Mammography images.

\section{Introduction}

Different fields, such as astronomy, medical imaging, and computer vision manage data of large volume. So, this data should be compressed to optimize the storage devices. There exist a lot of approaches in signal compression. Here, we present Wavelet-based techniques for compression procedures focusing on different threshold rules. The basic idea behind these techniques is to use Wavelets to transform data set into a different basis, where the non important information can be eliminated. Also, we have tested as classical, as novel Wavelet algorithms based on Atomic Functions, which present excellent compression results. Below, decimated Wavelet Transforms (WT) and the MAE fidelity criterion are used to evaluate the different compression methods for US and MG images.

Investigating the key properties of the different Wavelets we can justify the obtained experimental results in the compression of US and MG images.

\subsection{Wavelet Transform and Filter Banks}

The Discrete Wavelet Transform (DWT) is easy to realize using filter banks [1]. DWT can be implemented applying some equations, but it is usually made using filter 
bank techniques. The most popular scheme of the DWT for 2-D signal applies only two filters for rows and columns, as in the symmetric filter bank.

In tests carried out previously, it was found that better results are obtained when compressing the Ultrasound images with the Symlet Wavelet, and the Mammography images with the Daubechies Wavelet [2].

Based on this fact, we realize an evaluation to compare the acting of three Wavelet families based on Atomic Functions with the Wavelets that presented better acting. We use the complex Kravchenko-Rvachev wavelets $\psi(t)$ based on the Atomic Functions $u p(t), f_{u p}(t)$, and $\operatorname{eup}(t)[3]$.

\subsection{Compression by Wavelet Threshold}

The three main steps of compression using the Wavelet coefficient and threshold technique are as follows:

1. Calculate the Wavelet coefficient matrix applying WT to the original image.

2. Modify (threshold or shrink) the detail coefficients to obtain the reduced number of coefficients.

3. Encode the modified coefficients to obtain the compressed image.

In the two-level sub-bands decomposition, the coefficients on the first level are grouped into the vertical details $\left(\mathrm{LH}_{1}\right)$, horizontal details $\left(\mathrm{HL}_{1}\right)$, diagonal details $\left(\mathrm{HH}_{1}\right)$, and approximations $\left(\mathrm{LL}_{1}\right)$ sub-bands. The approximations part is then similarly decomposed in second level sub-bands. The directions reflect the order, in which the high-pass (H) and low-pass (L) filters of the WT are applied along the two dimensions of the original image.

\subsection{Thresholding Functions}

The thresholding functions [4] determine how the thresholds are applied to data. The most popular are four thresholds, a single threshold $( \pm t)$ is required for hard $\left.{ }_{[} \delta_{t}^{H}(w)\right]$, soft [ $\delta_{t}^{S}(w)$ ], and garrote [ $\delta_{t}^{G}(w)$ ] functions, but for semisoft function $\left[\delta_{t_{1}, t_{2}}^{S S}(w)\right]$, there are require two thresholds $\left( \pm \mathrm{t}_{1}\right.$ and $\left.\pm \mathrm{t}_{2}\right)$. Hard function does not modify the original data of the Wavelet coefficients, so for this reason we use only hard function in the experiments. The hard function is given below.

$$
S=\left\{\begin{array}{lll}
x & \text { si } & |x|>t \\
0 & \text { si } & |x| \leq t
\end{array} .\right.
$$

where $\mathrm{x}$ is the original signal, $\mathrm{S}$ is the thresholding signal and $\mathrm{t}$ is threshold.

\section{Wavelet Key Properties}

The Wavelet decomposition algorithm uses two analysis filters $\tilde{H}(z)$ (lowpass) and $\widetilde{G}(z)$ (highpass). The reconstruction algorithm applies the complementary synthesis 
filters $\mathrm{H}(\mathrm{z})$ (lowpass) and $\mathrm{G}(\mathrm{z})$ (highpass). These four filters constitute a perfect reconstruction filter bank. In the present case, the system is entirely specified by the lowpass filters $\mathrm{H}(\mathrm{z})$ and $\widetilde{H}(z)$, which form a biorthogonal pair. The highpass operators are obtained by simple shift and modulation and shown below.

$$
\widetilde{G}(z)=z H(-z) \text { and } G(z)=z^{-1} \widetilde{H}(-z) \text {. }
$$

For all the Wavelet families used in the compression scheme the following properties were obtained to justify the experimental results.

Frequency Response. This characteristic allows determining the behavior of the analysis and synthesis filters in a graphic way to appreciate the differences that there are among different Wavelet families used.

Approximation Order. This determines the number $\mathrm{L}$ of factors $\left(1+\mathrm{z}^{-1}\right)$ that divide the transfer function $H(z)$. The approximation order plays a crucial role in Wavelet theory [5]. It implies that the scaling function $\varphi(x)$ reproduces all polynomials of degree lesser or equal to $n=L-1$; in particular, it satisfies the partition of unity $\left(\sum_{k} \varphi(x-k)=1\right)$.

They are also directly responsible for the vanishing moments of the Wavelet analysis: $\int x^{n} \widetilde{\psi}(x) d x=0$ for $n=0,1,2, \ldots, L-1$. Finally, the order $L$ also corresponds to the rate of decay of the projection error as a scale how it goes to zero [6].

The next point concerns the stability of the wavelet representation and of its underlying multi-resolution bases. The crucial mathematical property is the translation of the scaling functions and Wavelets in Riesz bases [7]. Thus, one needs to characterize their Riesz bounds and other related quantities.

Let define a biorthogonal pair $\{\varphi, \varnothing\}$ as a set of scaling functions for which the cross-correlation filter is identity $\left(a_{\varphi, \phi}(\omega)=1\right)$. Below we mostly consider the autocorrelation filter $a_{\varphi, \varphi}(\omega)$ denoted as $a_{\varphi}(\omega)$.

Riesz Bounds. The tightest upper and lower bounds, $B<1$ and $A>0$, of the autocorrelation filter of $\varphi(x)$ are the Riesz bounds of $\varphi(x)$ that are given by.

$$
A=\inf _{c \in \ell^{2}} \frac{\left\|\sum_{k \in \mathrm{Z}} c_{k} \varphi(x-k)\right\|_{L^{2}}}{\|c\|_{\ell^{2}}} \text { and } B=\sup _{c \in \ell^{2}} \frac{\left\|\sum_{k \in \mathrm{Z}} c_{k} \varphi(x-k)\right\|_{L^{2}}}{\|c\|_{\ell^{2}}} .
$$

The existence of the Riesz bounds ensures that the underlying basis functions are in $L^{2}$ and that coefficients of transform are linearly independent in the $\ell^{2}$ space. The Riesz basis property expresses equivalence between the $L^{2}$-norm of the expanded functions and the $\ell^{2}$-norm of their coefficients in the Wavelet or scaling function basis. There is a perfect norm equivalence (Parseval's relation), if and only if $A=B=$ 1 , and in this case the basis is orthonormal.

Projection Cosine. The (generalized) projection angle $\theta$ between the synthesis and analysis subspaces $V_{a}$ and $\tilde{V}_{a}$ is defined as [8]: 


$$
\cos \theta=\inf _{f \in \widetilde{V}_{a}} \frac{\left\|\mathrm{P}_{a} f\right\|_{L^{2}}}{\|\left. f\right|_{L^{2}}}=\frac{1}{\sup _{\omega \in[0,2 \pi]} \sqrt{a_{\varphi}(\omega) \cdot a_{\tilde{\varphi}}(\omega)}} .
$$

This fundamental quantity is scale-independent, it allows comparing the performance of the biorthogonal projection $\widetilde{\mathrm{P}}_{a}$ with that of the optimal least squares solution $\mathrm{P}_{a}$ for a given approximation space $V_{a}$. Specifically, we have the following sharp error bound [9]:

$$
\forall f \in L^{2},\left\|f-\mathrm{P}_{a} f\right\|_{L^{2}} \leq\left\|f-\widetilde{\mathrm{P}}_{a} f\right\|_{L^{2}} \leq \frac{1}{\cos \theta}\left\|f-\mathrm{P}_{a} f\right\|_{L^{2}} .
$$

The projection angle $\theta$ between the synthesis and analysis subspaces should be 90 degrees in orthogonal spaces. In other words, the biorthogonal projector $\mathrm{P}_{a}$ will be essentially as good as the optimal one (orthogonal projector onto the same space) when the value $\cos \theta$ is close to one.

\subsection{Criteria of Fidelity}

To evaluate in objective manner the fidelity of the compressed images we apply several criteria that usually are used the difference between two images [10].

Mean Absolute Error (MAE). This criterion is often applied as a numeric measure of distortion for fine details and contours of the image and characterizes the average difference between the original and compressed images.

$$
M A E=\frac{1}{M \cdot N} \sum_{m=1}^{M} \sum_{n=1}^{N}|y[m, n]-\hat{y}[m, n]|
$$

where: $M, N$ are size of the image. $y[m, n]$ is original image; $\hat{y}[m, n]$ is compressed image.

Compression Rate (CR). This criterion characterizes the compression quantity. It given by the following equation:

$$
C R=\frac{\text { Original image size }}{\text { Compressed image size }} .
$$

\section{Simulation Results}

We carried out numerous simulated experiments to compare the performance of the compression algorithm using different Wavelet functions (classical and based in AFs). Firstly, let present the experimental results applying Symlet filters for Ultrasound images, and, secondly, we present the experimental results applying Daubechies filters for Mammography images. Both results are compared with three different families of Wavelet filters based en AFs. We use five decomposition levels in the compression procedure. Visual results are not presented only the objective evaluation. 

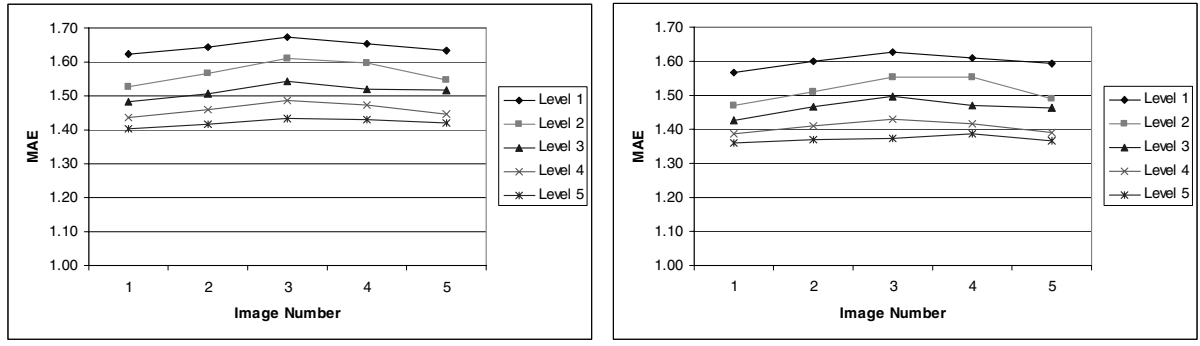

Fig. 1. MAE criterion for compressed Ultrasound images with Symlet filters and based on AF $u p(t)$ Wavelet filters, respectively (left to right)
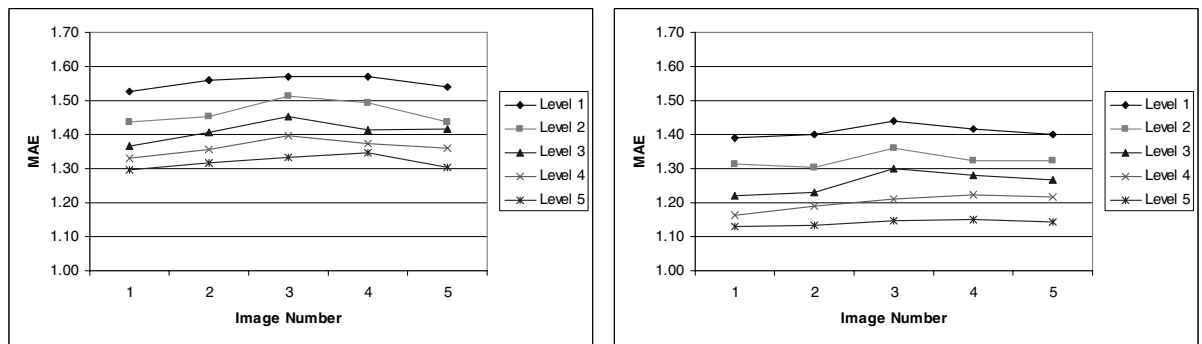

Fig. 2. MAE criterion for compressed Ultrasound images with based on $\mathrm{AF} f u p_{2}(t)$ and eup(t) Wavelet filters, respectively (left to right)
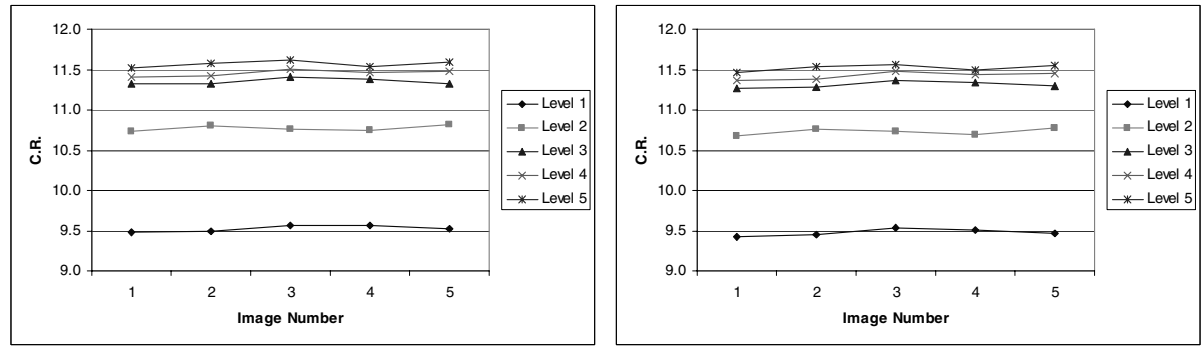

Fig. 3. CR criterion for compressed Ultrasound images with Symlet filters and based on AF $u p(t)$ Wavelet filters, respectively (left to right)
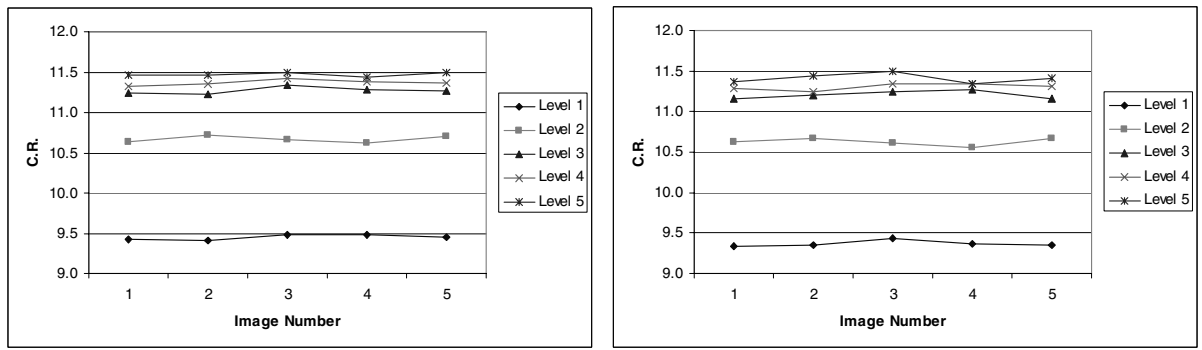

Fig. 4. CR criterion for compressed Ultrasound images with based on $\mathrm{AF} f u p_{2}(t)$ and eup $(t)$ Wavelet filters, respectively (left to right) 

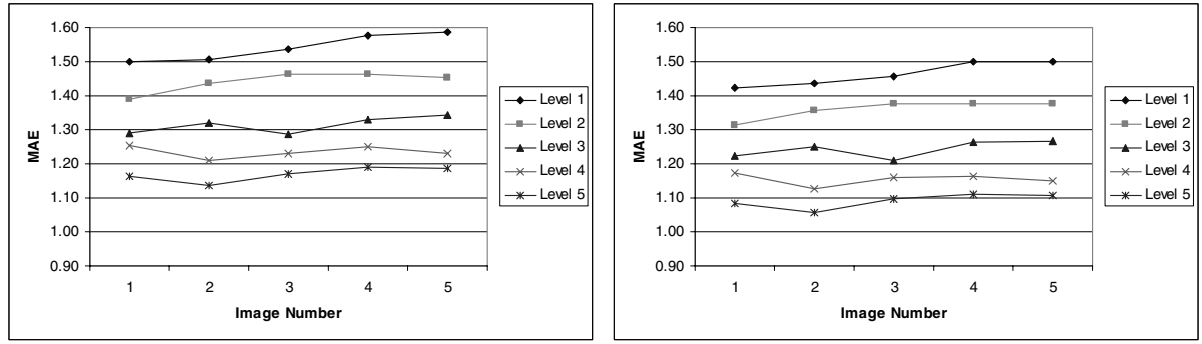

Fig. 5. MAE criterion for compressed Mammography images with Daubechies filters and based on $\mathrm{AF} u p(t)$ Wavelet filters, respectively (left to right)
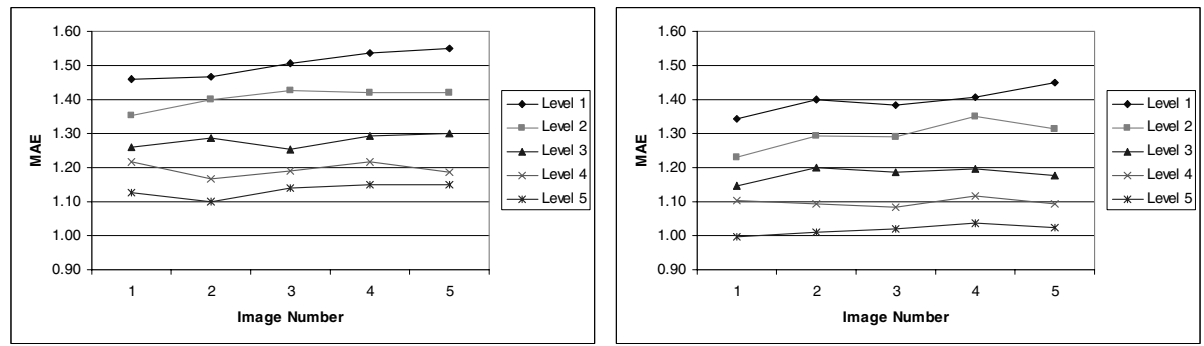

Fig. 6. MAE criterion for compressed Mammography images with based on $\operatorname{AF} \operatorname{fup}_{2}(t)$ and $\operatorname{eup}(t)$ Wavelet filters, respectively (left to right)
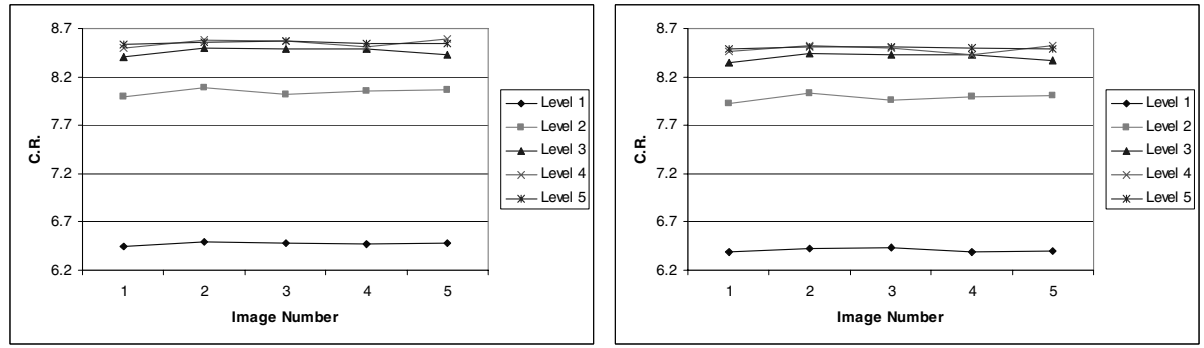

Fig. 7. CR criterion for compressed Mammography images with Daubechies filters and based on $\mathrm{AF} u p(t)$ Wavelet filters, respectively (left to right)
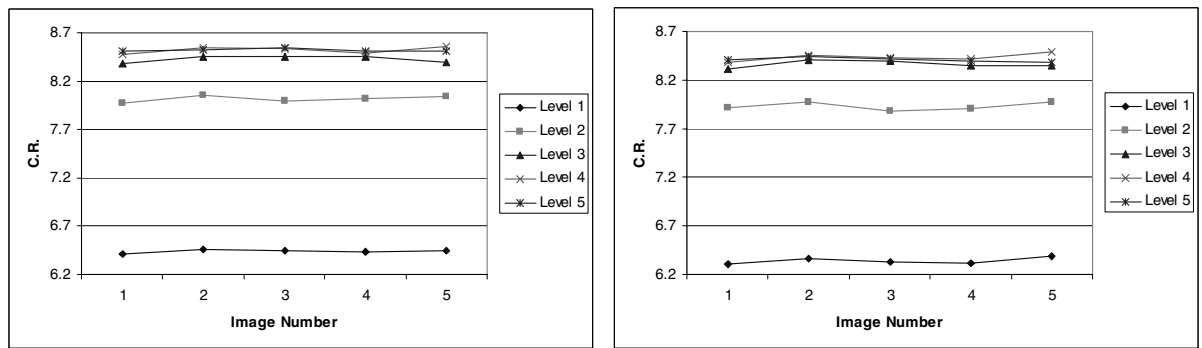

Fig. 8. CR criterion for compressed Mammography images with based on $\mathrm{AF} f u p_{2}(t)$ and eup $(t)$ Wavelet filters respectively (left to right) 
Finally, the table 1 presents the key properties of the different Wavelets used in compression of the US and MG images.

Table 1. Summary of key properties for different Wavelet filters

\begin{tabular}{|c|c|c|c|c|c|c|c|c|c|c|c|c|}
\hline \multicolumn{13}{|c|}{ Key properties for different Wavelet filters. } \\
\hline \multirow[t]{2}{*}{ Type } & \multicolumn{2}{|c|}{ Wavelet 9/7 } & \multicolumn{2}{|c|}{ Daubechies 8} & \multicolumn{2}{|c|}{ Symlet 8} & \multicolumn{2}{|c|}{ A.F.W. $u p(t)$} & \multicolumn{2}{|c|}{ A.F.W. $f u p_{2}(t)$} & \multicolumn{2}{|c|}{ A.F.W. $\operatorname{eup}(t)$} \\
\hline & Dec. & Rec. & Dec. & Rec. & Dec. & Rec. & Dec. & Rec. & Dec. & Rec. & Dec. & Rec. \\
\hline $\begin{array}{l}\text { Approxima } \\
\text { tion Order }\end{array}$ & \multicolumn{2}{|c|}{4} & \multicolumn{2}{|c|}{4} & \multicolumn{2}{|c|}{4} & \multicolumn{2}{|c|}{4} & \multicolumn{2}{|c|}{4} & \multicolumn{2}{|c|}{4} \\
\hline $\begin{array}{c}\text { Projection } \\
\text { cosine }\end{array}$ & \multicolumn{2}{|c|}{0.98387} & \multicolumn{2}{|c|}{0.98879} & \multicolumn{2}{|c|}{0.98781} & \multicolumn{2}{|c|}{0.99176} & \multicolumn{2}{|c|}{0.99472} & \multicolumn{2}{|c|}{0.99769} \\
\hline \multirow{2}{*}{$\begin{array}{l}\text { Riesz } \\
\text { Bounds }\end{array}$} & 0.926 & 0.943 & 0.833 & 0.849 & 0.880 & 0.896 & 0.792 & 0.806 & 0.713 & 0.726 & 0.641 & 0.653 \\
\hline & 1.065 & 1.084 & 1.267 & 1.290 & 1.273 & 1.295 & 1.514 & 1.542 & 1.802 & 1.834 & 2.145 & 2.183 \\
\hline
\end{tabular}

It is known from statistical theory that the approximation property of estimation of random variable can be given in form of relative error $\delta=2(1-r)$, where $\mathrm{r}$ is correlation coefficient that is equal to projection cosine in this case. So, calculations of this error using table 1 show that Wavelet based on eup $(x)$ can produce relative variance error of 0,00464 (6.8\% in RMS value), but in same time Wavelet Daubechies 8 gives value of 0,02242 (more than $15 \%$ in RMS value), and Wavelet $9 / 7$ value of 0,03234 (more than $18 \%$ in RMS value). So, Wavelet based on eup $(x)$ function gives about three less a relative error in RMS values than Wavelet 9/7 used in JPEG2000 standard.

\section{Conclusions}

There were carried out numerous tests in comparing of different Wavelets functions to choose the best one for compression of medical US and MG images. It is observed that for two modalities of images used in the tests the Wavelets families based on atomic functions presented smaller levels of mean absolute error, with relationship to their equivalent of the Daubechies and Symlet families. The compression level also decreased lightly but better image quality is conserved.

The Wavelet family based on the atomic function eup $(x)$ present better levels of MAE for two image modalities. It is observed in the carried out analysis that Daubechies and Symlet filters are most selective than the Wavelet 9/7 used in standard JPEG2000. The filters of the Wavelet based on the atomic function eup $(x)$ are presented the best frequency response. One can see that all the used filters have the same approximation order. This derives mainly in two things, the Wavelet filters have the same number of coefficients, and therefore, they imply the same computational complexity when being implemented in the compression algorithms, and the convergence of the error will be of the same order for all the filters.

The existence of the limits Riesz bounds demonstrates that the coefficients of the analysis and synthesis filters are lineally independent. The projection cosine measure shows that the families of Wavelets based on atomic functions are near to optimal 
ones; this implies that they are "better" orthogonal and "most independent". Also, the fact that they are lineally independent assures that errors are hardly presented to the decomposition/reconstruction procedure. Likewise the different properties of the Wavelet filters found here demonstrates that the filters of the Wavelets families based on atomic functions can realize the approximation better in comparison with traditional Wavelets, potentially giving superior quality of compression, guaranteeing the same level of error.

Acknowledgments. This work is supported by CONACyT and National Polytechnic Institute of Mexico.

\section{References}

1. Jähne, B.: Practical Handbook on Image Processing for Scientific and Technical Applications. CRC Press, Boca Raton (2004)

2. Sanchez, J.L., Ponomaryov, V.: Wavelet Compression Applying Different Threshold Types for Ultrasound and Mammography Images. GESTS Intern. Trans. on Computer Sciences and Engineering 39(1), 15-24 (2007)

3. Yu, G., Kravchenko, V., Pustovoit, V.: A New Class of WA-Systems of KravchenkoRvachev Functions. Doklady Mathematics 75(2), 325-332 (2007)

4. Jan, J.: Medical Image Processing, Reconstruction and Restoration: Concepts and Methods. Taylor \& Francis/ CRC Press (2006)

5. Vetterli, M., Kovacevic, J.: Wavelets and Subband Coding. Prentice-Hall, Englewood Cliffs (1995)

6. Villemoes, L.: Wavelet analysis of refinement equations. SIAM J. Math. Anal. 25(5), 1433-1460 (1994)

7. Meyer, Y.: Ondelettes, Hermann, Paris, France (1990)

8. Unser, M., Aldroubi, A.: A general sampling theory for nonideal acquisition devices. IEEE Trans. Signal Process. 42(11), 2915-2925 (1994)

9. Strang, G., Nguyen, T.Q.: Wavelets and Filter Banks. Wellesley-Cambridge Press, Cambridge (1996)

10. Ponomaryov, V., Sanchez-Ramirez, J.L., Juarez-Landin, C.: Evaluation and Optimization of the Standard JPEG2000 for Compression of Ultrasound Images. Telecommunications and Radio Engineering 65(11), 1005-1017 (2006) 